\title{
Dientes neonatales: Reporte de un caso y revisión de la literatura
}

\section{Neonatal teeth: A case report and review of literature}

\section{Souza ACRA*, Normandia CS**, Melo LT**, López Alvarenga R***, Souza LN****}

\section{RESUMEN}

Los dientes natales y neonatales son anomalías relativamente raras en los recién nacidos, que pueden causar trastornos en la alimentación, ulceraciones traumáticas sublinguales (enfermedad de Riga-Fede) y preocupaciones en cuanto a la aspiración de los mismos. La diferencia entre los dientes natales y neonatales está en el momento de la erupción. Los dientes natales son dientes que aparecen en la cavidad oral en el momento del nacimiento y los dientes neonatales son los que aparecen durante las primeras semanas de vida. El objetivo de este artículo es presentar a través de una revisión de la literatura, los aspectos más relevantes de los dientes neonatales y la presentación de un caso clínico de un recién nacido con pocos días de vida con diente neonatal, donde el tratamiento aplicado fue la extracción.

Palabras clave: Dientes neonatales, dientes natales, odontopediatría, bBebés, anomalías dentarias.

\section{SUMMARY}

Natal and neonatal teeth are rare anomalies in newborns that can cause feed disturbances, tongue ulceration (Riga-Fede disease) and risk of aspiration of them. The difference between natal and neonatal teeth is based on time of eruption. The natal teeth are those that are already present in the oral cavity since birth, and neonatal teeth are those that appear in the oral cavity in the first month of life. The aim of this paper is to present a case of neonatal tooth as well as to discuss, with literature basis, the most relevant aspects concerning the diagnosis and treatment employed.

Key words: Neonatal teeth, natal teeth, pediatric dentistry, infants, dental anomalies.

Fecha de recepción: 19 de noviembre de 2009.

Aceptado para publicación: 10 de diciembre de 2009.

* Prof. Adjunta de Odontopediatría y Ortodoncia, Centro Universitario Newton Paiva, Belo Horizonte. Brasil.

** Alumnas del Curso de Odontología do Centro Universitario Newton Paiva, Belo Horizonte. Brasil.

*** Licenciado en Odontología por la universidad del Centro Universitario Newton Paiva, Belo Horizonte. Brasil.

**** Prof. Adjunto de Cirugía Oral y Maxilofacial, Centro Universitario Newton Paiva, Belo Horizonte. Brasil.

Souza ACRA, Normandia CS, Melo LT, López Alvarenga R, Souza LN. Dientes neonatales: Reporte de un caso y revisión de la literatura. Av. Odontoestomatol 2011; 27 (5): 253-258. 


\section{INTRODUCCIÓN}

La evolución del sistema estomatognático de los niños está directamente relacionada con el desarrollo de otros sistemas, por lo que es de suma importancia conocer las características de normalidad y alteraciones clínicas que pueden ocurrir, siendo que algunas de estas características son efímeras y solamente se encuentran en esta fase de la vida $(1,2)$.

Una de las características relevantes es la erupción de los incisivos centrales inferiores deciduos, que son los primeros dientes que erupcionan en la cavidad oral, aproximadamente a los seis meses de vida del niño (3). Sin embargo, hay relatos de casos de niños que nacen con dientes erupcionados en la cavidad oral o que erupcionan en el primer mes de vida. Estos dientes pueden ser llamados de dientes natales y neonatales respectivamente y pueden pertenecer a la dentición normal o supernumerarios (4-9).

Los factores etiológicos más comunes son la posición superficial del germen dental en desarrollo, hereditariedad, síndromes congénitos y fisura labiopalatina (4). La región más afectada es la anterior de la mandíbula y los incisivos inferiores son los dientes más comúnmente afectados. Los dientes natales o neonatales erupcionados pueden presentar hipermovilidad con riesgo de desprendimiento seguido de una deglución o aspiración del diente. Otra complicación son las lesiones secundarias tanto en el pecho materno durante la lactancia o lesiones en base en la lengua conocidas como Ulcera de Riga-Fede, dificultando la lactancia materna (4,6-10).

El tratamiento para esta anomalía es un asunto que se debería de estar bien estudiado por los profesionales involucrados antes de decidirse por la extracción o el mantenimiento del diente en la cavidad oral. Varios aspectos deben ser tenidos en cuenta antes de la medida terapéutica, como la preservación del espacio de la arcada dentaria, el estado funcional y qué diente está afectado (supernumerarios o normales), evitando una extracción iatrogénica (4).

La finalidad del siguiente trabajo es presentar un caso de diente neonatal y realizar una revisión de la literatura sobre los aspectos más importantes en cuanto a diagnóstico, etiología, epidemiología, característi- cas clínicas, radiográficas y medidas terapéuticas más adecuadas para los dientes neonatales.

\section{CASO CLÍNICO}

Recién nacido de sexo masculino con 40 días de edad, fue remitido a la clínica Odontológica del Centro Universitario Newton Paiva por su médico Pediatra, por presentar un diente neonatal en la región anterior de la mandíbula que había erupcionado hace 15 días.

Su madre refiere que el diente estaba traumatizando su pecho e impidiendo la lactancia. Además, el diente presentaba una extrema movilidad con riesgo de desprendimiento y aspiración de la pieza dentaria de acuerdo con el médico Pediatra (Figs. 1 y 2).

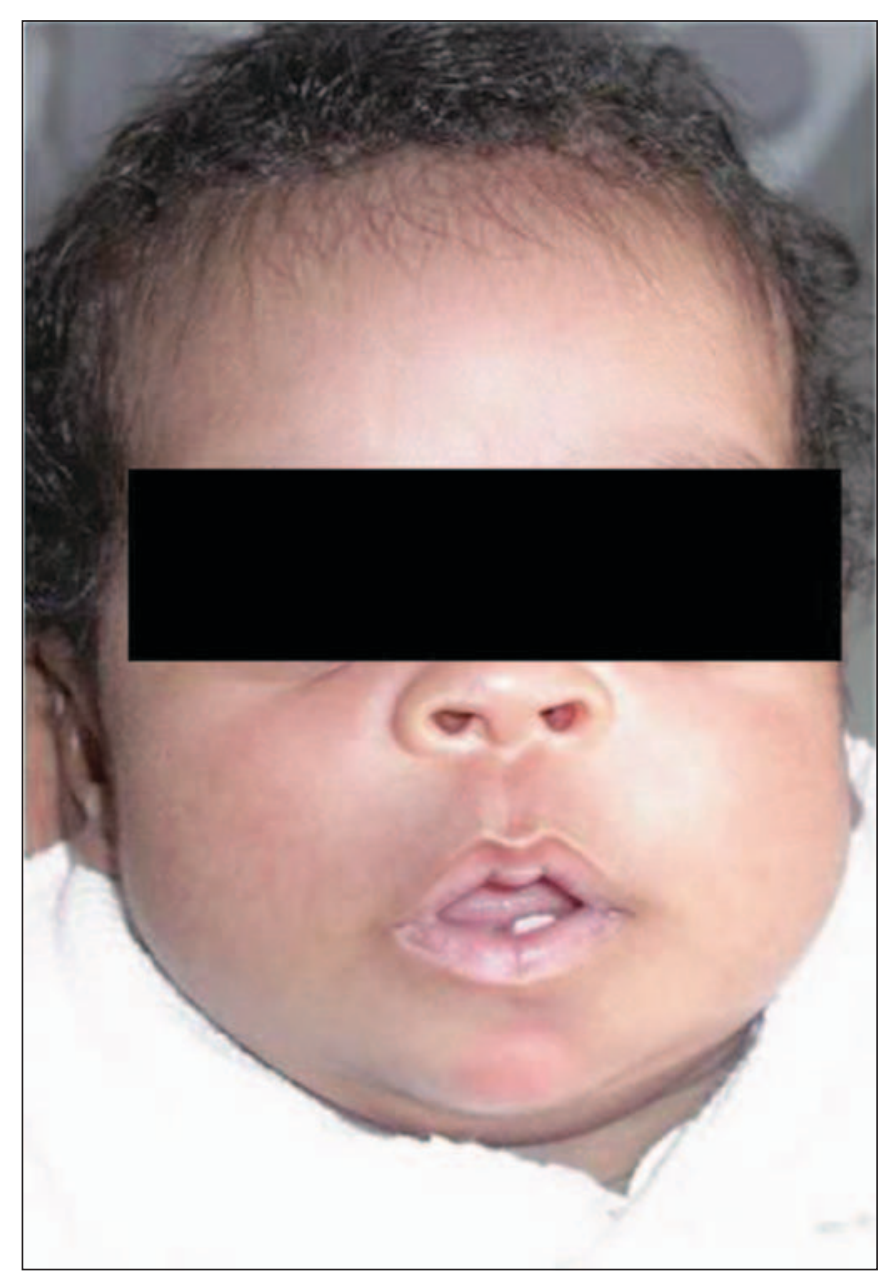

Fig. 1. Vista panorámica en la que se aprecia el diente neonatal. 


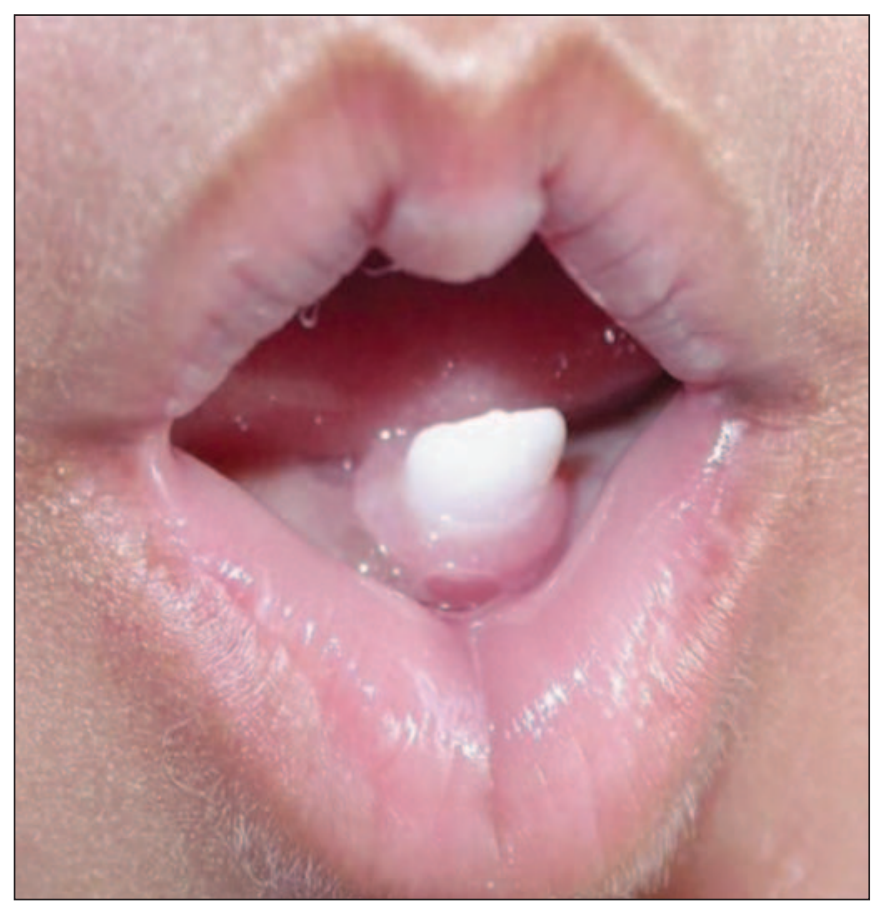

Fig. 2. Vista directa intraoral donde se observa el diente neonatal.

Al realizar el interrogatorio a la madre sobre los antecedentes médico personales y familiares, relató que el bebé nació sin complicaciones, parto normal, a término y sin ninguna evidencia de enfermedades sistémicas, anomalía congénita, o trastornos en el desarrollo.

Durante la exploración intraoral reveló una estructura semejante a un diente en la zona central del reborde de la mandíbula, que presentaba gran movilidad y no se observó ninguna ulceración en la lengua (enfermedad de Riga-Fede) y sí un discreto eritema provocado por el trauma constante de la lengua en el diente, que estaba ligeramente vestibulizado.

Debido la dificultad de lactancia, la gran movilidad del diente y al alto riesgo de desprendimiento, se decide por la extracción preventiva de la pieza dentaria la cual es retirada sin dificultad y sin provocar complicaciones posteriores al recién nacido. El material utilizado para la extracción fue solamente una gaza y anestésico tópico debido la movilidad del mismo.

Después de la extracción se comprobó que el diente no tenía la formación radicular (figs. 3 y 4). El segui-

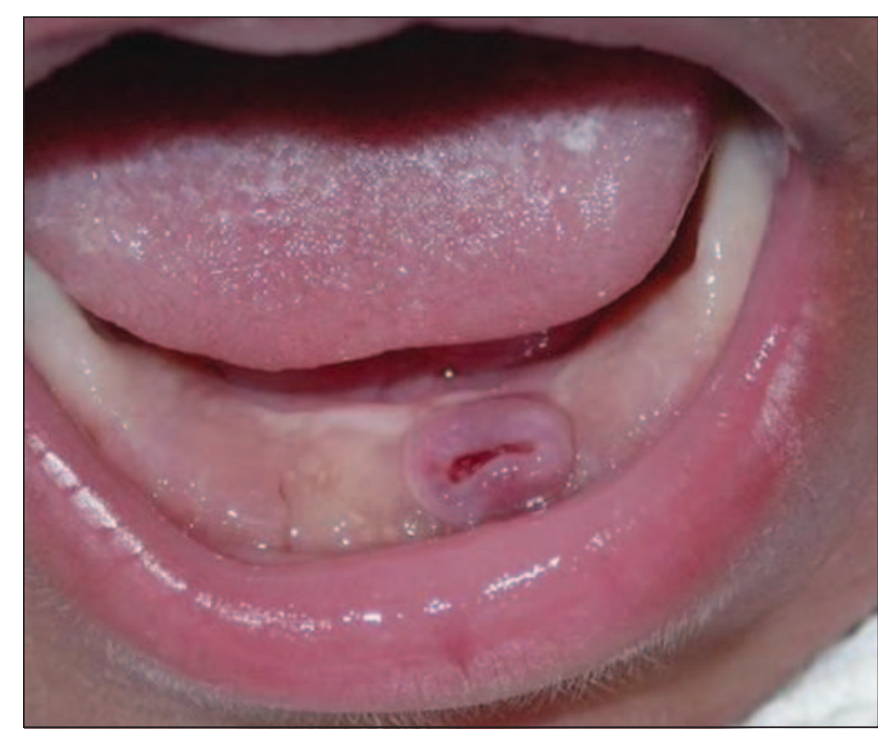

Fig. 3. Vista directa intraoral exhibiendo el aspecto de la región del diente extraído.

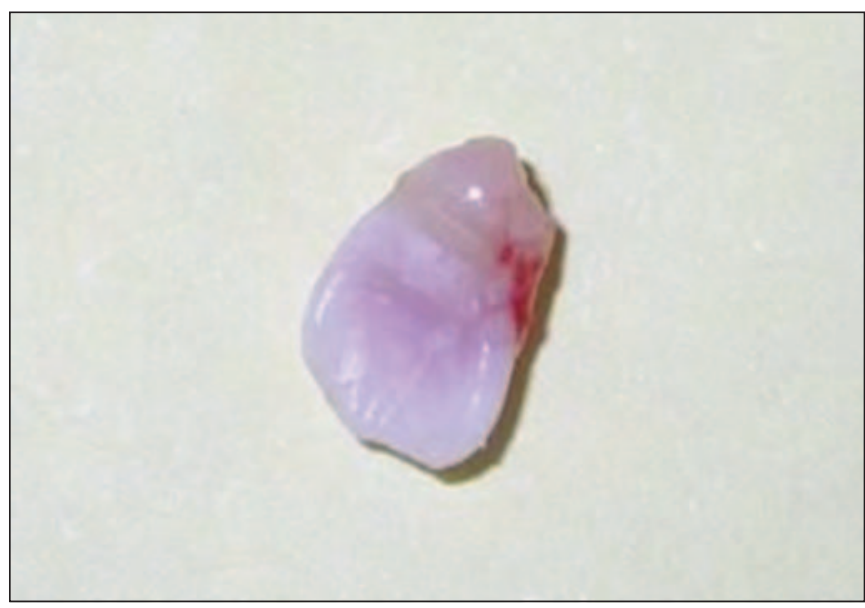

Fig. 4. Pieza dentaria tras la extracción.

miento del paciente con controles periódicos mostró en 6 meses una erupción normal de los incisivos deciduos inferiores, configurando un cuadro de diente neonatal extranumerario.

\section{DISCUSIÓN}

Varios términos han sido utilizados para designar a los dientes que han erupcionado antes del tiempo normal, tales como diente congénito, diente fetal, diente predeciduo, diente prematuro, diente connatal 
y diente precoz. A comienzos de los años cincuenta Massler y Savara clasificaron los dientes que erupcionan prematuramente, de este modo los dientes presentes al nacimiento fueron llamados de dientes natales, mientras que aquellos que erupcionan durante las primeras semanas de vida fueron llamados de dientes neonatales. Esta terminología es la más aceptada en la literatura y utilizada en el presente. El caso presentado corresponde a un diente neonatal.

La frecuencia de aparición según algunos autores es de un caso por cada 1.100-3.700 nacimientos $(4,11,12)$. La mayoría de los estudios mostraron que 95\% de los casos de dientes natales y neonatales son de la serie normal y solamente el $5 \%$ son supernumerarios (12). Otro dato relevante es que esta alteración es más frecuente en niñas que en niños en una relación de 3 a $1(3,10)$. Los dientes que erupcionan precozmente con mayor frecuencia son los incisivos centrales inferiores y en un $60 \%$ de los casos relatados en la literatura pueden estar de forma bilateral y simétrica. En el caso presentado el diente neonatal era supernumerario, siendo que 6 meses tras la extracción ocurrió la erupción normal de los incisivos deciduos inferiores.

Con el paso del tiempo se han hecho muchas sugerencias con respecto a la causa de la erupción prematura, incluyendo hipovitaminosis, estados febriles, estimulación hormonal, pielitis, disturbios en la gestación, granuloma piógeno, posición superficial del germen dental y sífilis congénita $(4,3,13)$. Varios autores han citado una asociación frecuente con antecedentes familiares, así como también con múltiples síndromes y anormalidades del desarrollo como Displasia Ectodérmica y Condroectodermal, Oculomandibulodiscefalia, Paquioniquia Congénita, Síndrome de Pierre Robin, Síndrome de WiedemannRautenstrauch y Síndrome de Paladar Hendido o Labio Hendido $(8,14)$. En el caso presentado se verificó una posición superficial del germen dental, sin antecedentes sindrómicos / familiares.

Generalmente, los dientes natales y neonatales tienen una gran movilidad debido la poca estructura radicular, que indica un alto riesgo de deglución o aspiración del diente $(4,13,15)$ para el recién nacido y que, corresponde con las características del caso presentado. El esmalte de estos dientes tiene una formación frágil o se muestra con una espesura más fina de lo normal.

Los dientes natales y neonatales primarios son similares a los dientes normales primarios, pero la mayoría de las veces son pequeños, cónicos, amarillentos, con esmalte y dentina hipoplásica $(3,7,10,11)$. En el caso presentado el diente neonatal es semejante a un diente deciduo y puede ser clasificado como diente de corona sólida perdida unida al alvéolo por la mucosa oral; pequeña o ninguna raíz, de acuerdo con la clasificación descrita en la literatura (16).

En diversos casos, los dientes natales y neonatales pueden provocar úlceras traumáticas en la superficie ventral de la lengua, la cual se conoce como enfermedad de Riga-Fede o en el pecho materno. La enfermedad de Riga-Fede es un proceso inflamatorio poco frecuente y benigno caracterizado por una ulceración ubicada en la parte ventral de la lengua, que está causada por el trauma repetido sobre los incisivos inferiores de los dientes natales y neonatales que ocurre durante la lactancia y el reflejo instintivo de succión $(3,9,13)$.

El diagnóstico diferencial más importante se obtiene mediante una radiografía para diferenciar un diente de la serie normal de un supernumerario para evitar exodoncias indiscriminadas $(4,16)$. Además, el examen clínico minucioso y bien realizado es de fundamental importancia para un correcto diagnóstico, verificando el grado de movilidad del diente y presencia de borde incisal filoso que puede provocar lesiones en la superficie ventral de la lengua o en el pecho materno (4). En nuestro caso presentado se observó una vestibulización y movilidad del diente neonatal, dificultades en la lactancia materna y eritema provocado por el trauma constante de la lengua en el diente. Teniendo en cuenta los hallazgos clínicos y la falta de cooperación del paciente se optó por no hacer examen radiográfico antes de la extracción.

Otra alternativa de tratamiento para los dientes natales y neonatales consiste en desgastar los bordes de los incisivos con una piedra de diamante de grano fino o recubrimiento de los bordes con resina compuesta, para evitar que se lesione a sí mismo o a la madre en la lactancia. Cuándo se indica esta con- 
ducta, se debe explicar a los padres del recién nacido la importancia de la conservación para garantizar la integridad de la dentición decidua $(4,9,11)$. En el caso de nuestro paciente no había la posibilidad de aplicar este tratamiento debido la gran movilidad del diente.

Cuando el tratamiento elegido es la extracción es aconsejable esperar a que el recién nacido tenga por lo menos de 10 a 14 días de vida, esto permitirá que la flora intestinal del lactante produzca vitamina K la cual es esencial para los niveles adecuados de protrombina que interviene en los procesos de coagulación (17-19). En el caso presentado el paciente ya tenía 40 días de vida y los niveles de vitamina K estaban dentro de los límites normales.

\section{CONCLUSIONES}

La revisión de la literatura y el caso clínico descrito permiten realizar las siguientes consideraciones:

- A pesar de que esta anomalía es relativamente poco frecuente en los recién nacidos es una de las más prevalentes, afectando principalmente a la región de los incisivos;

- La decisión de extraer o mantener el diente neonatal dependerá de los hallazgos clínicos, debe tenerse en cuenta el comprometimiento de la función, la movilidad del diente, el riesgo de aspiración y de lesión en la lengua del bebé o del pecho de la madre;

- La cavidad oral del recién nacido tiene algunas características peculiares de esta fase de la vida, por lo tanto, es de extrema importancia conocer la cavidad oral en su conjunto y su normalidad, para que se haga un diagnóstico correcto de las anomalías del desarrollo y decidir el tratamiento más adecuado para cada caso.

\section{BIBLIOGRAFÍA}

1. Baldani MH, Lopes CMDL, Scheidt WA. Prevalência de alterações bucais em crianças atendidas nas clínicas de bebês públicas de Ponta Grossa-PR, Brasil. Pesqui Odontol Bras. 2001;15(4):302-7.
2. Santos FFC, Pinho JRO, Libério AS, Cruz MCFN. Prevalência de alterações orais congênitas e de desenvolvimento em bebês de 0 a 6 meses. Rev odonto ciênc. 2009;1(24):77-80.

3. Zhu J, King GD. Natal and neonatal teeth. Oral Health. 1996;86(6):41-8.

4. Yared FNFG, Yared KFG. Dentes Natais e Neonatais: Diagnóstico, Decisões de Tratamento e Atenção ao Traumatismo Dental Precoce. J Brás Odontopediatr Odontol Bebê. 2002;5(23): 21-7.

5. Ventiades FJ, Tattum BK. Patología oral del recién nacido. Rev. Soc. Boliv. Pediatr. 2006;45(2): 112-5.

6. Ferreira SH; Feldens EG, Cardoso L, Kramer PF, Müller A. Dente Neonatal Associado à Anquiloglossia em Recém-Nascido: Relato de Caso Clínico. Rev Ibero-am Odontopediatr Odontol Bebê. 2006;9(51/52):347-52.

7. Anegundi RT, Sudha P, Kaveri H, Sadanand K. Natal and neonatal teeth: A report of four cases. J Indian Soc Ped Pre Dent. 2002;3(20):8692.

8. Diniz MB, Gondim JO, Pansani CA, Lima FCBA. A importância da interação entre odontopediatras e pediatras no manejo de dentes natais e neonatais. Rev Paul Pediatr. 2008;26(5):64-9.

9. Guzmán A, Mendoza G. Dientes natales y enfermedad de Riga Fede. Dermatol Pediatr Lat. 2005;3(2):152-7.

10. Gómez VM. Dientes natales y neonatales, reporte de três casos: Segunda Parte. Acta Odontol. Venez. 1997;35(3).

11. Long SM, Chelotti A, Rego MA, Jorge AO. Dente natal: relato de caso clínico. Rev. Assoc Paul Cir Dent. 1994;48(2):1291-4.

12. Diaz PE, Podestá MCE. Dientes natales y neonatales: Enfermedad de Riga Fede. Vis Den. 2005; 8(6):1-4. 
13. Massler M, Savara S.B. Natal and neonatal teeth. A review of twenty - four cases reported in the literature. J Pediatr. 1950;36:349-59.

14. Neves ACC, Patrocínio MC, Leme KP, Ui RT. Anomalias Dentárias em Pacientes Portadores de Fissuras Labiopalatinas: Revisão de Literatura. Rev Biociênc. 2002;8(2):75-81.

15. Bonecker MJS. Dente natal e neonatal. Rev. APCD. 1997;51: 373-4.

16. Hebling J, Zuanon ACC, Vianna DR. Dente Natal: A case of natal teeth. Odontologia Clínica. 1997;7(1):37-40.

17. Rusmah M. Natal and neonatal teeth: a clinical and histological study. J.Clin. Pedodont. Dent. 1991;15(4):251-3.
18. Allwright WC. Natal and neonatal teeth: a study among Chinese in Hong Kong. Br Dent J. 1958; 105:163-72.

19. Berendsen WJH, Wakkerman HL. Continued growth of the dentinal papillae after extraction of neonatal teeth: report of case. Journal of dentistry for children. 1988;139-41.

\section{CORRESPONDENCIA}

Rodrigo López Alvarenga

C/ Gomera, 6, Esc. 2, 3 $\mathrm{H}$

04770 Adra. Almería

E-mail: mre_lopez@hotmail.com 